\title{
Verzeichnis der Tabellen und Graphiken
}

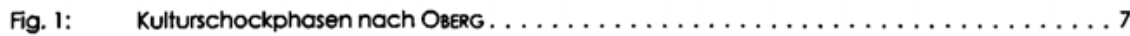

Fig. 2: Stufenmodell des Kulturschocks nach Adter. $\ldots \ldots \ldots \ldots \ldots \ldots \ldots \ldots \ldots \ldots$

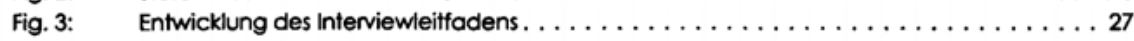

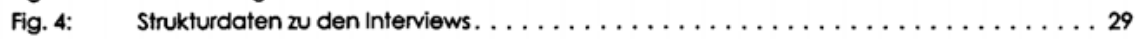

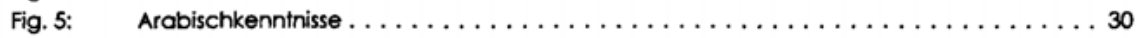

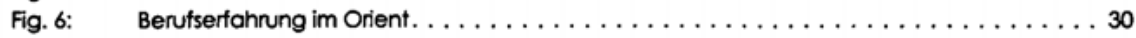

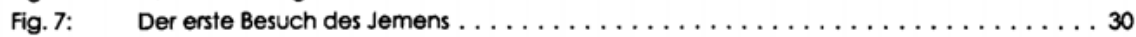

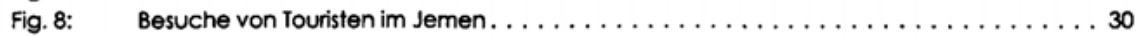

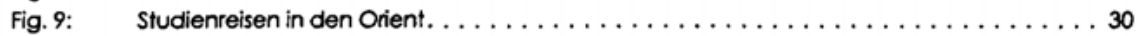

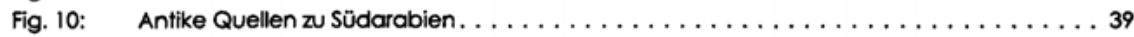

Fig. 11: Autoren von SÜdwestarabienberichten vor CARSTEN NIEBUHR $\ldots \ldots \ldots \ldots \ldots \ldots \ldots$ 51

Fig. 12: Autoren von Südwestarabienberichten seit CARSTEN NiEBUHR (bis 1899) $\ldots \ldots \ldots \ldots .62$

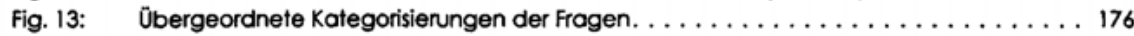

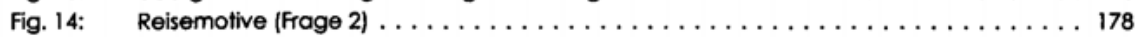

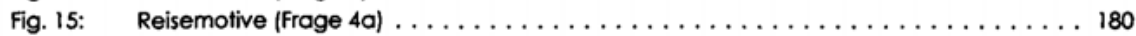

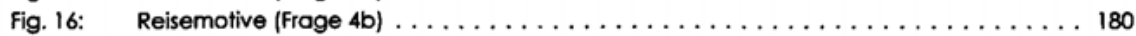

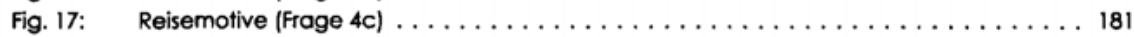

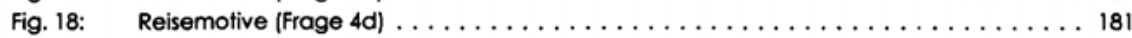

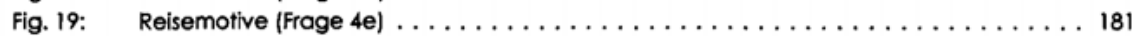

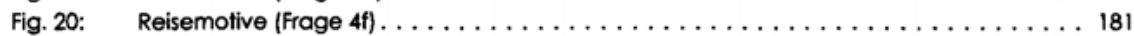

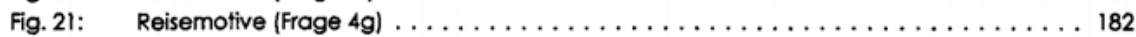

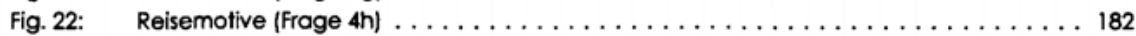

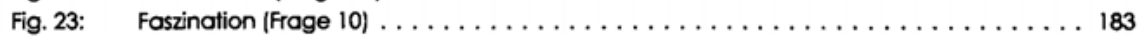

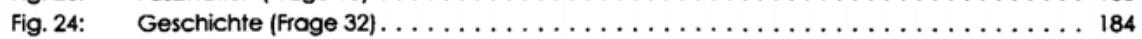

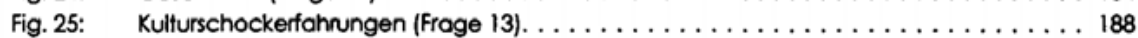

Fig. 26: Befremden (Frage 14) . . . . . . . . . . . . . . . . . . . . 189

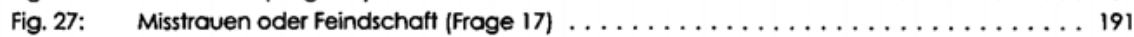

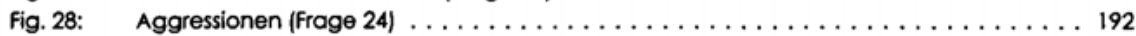

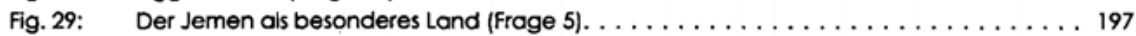

Fig. 30: Vorurteile Über Jemeniten (Frage 26) $\ldots \ldots \ldots \ldots \ldots \ldots \ldots \ldots \ldots \ldots \ldots \ldots \ldots$

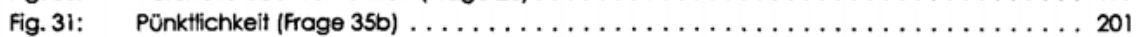

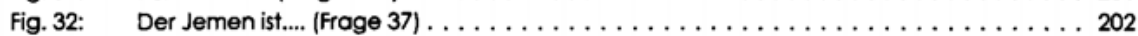

Fig. 33: Unterschiede zwischen Jemeniten und Europäern (Frage 12) . . . . . . . . . 203

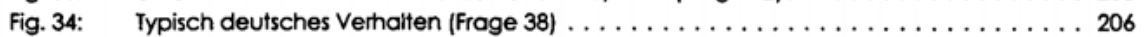

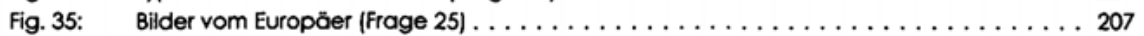

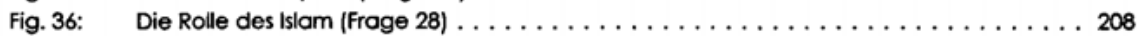

Fig. 37: Die Beobachtung von Beduinen (Frage 30) . . . . . . . . . . . . . . . . 209

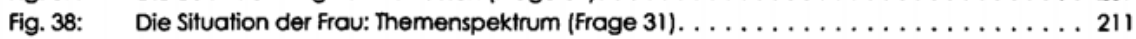

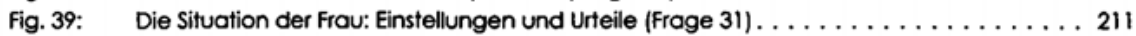

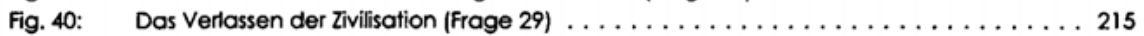

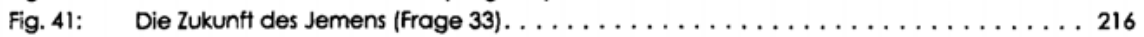

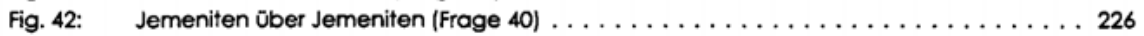

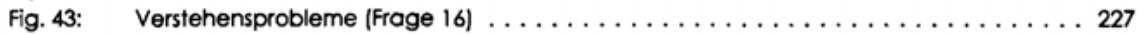

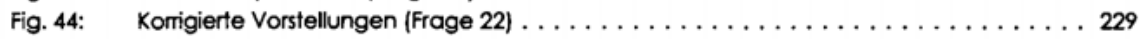

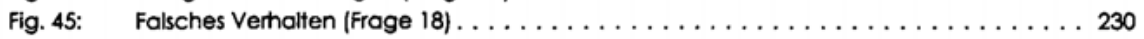

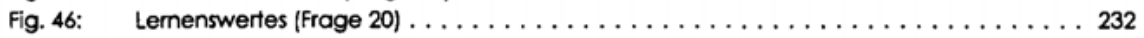

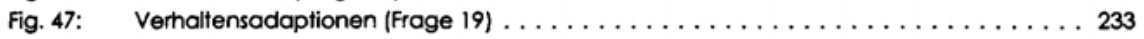

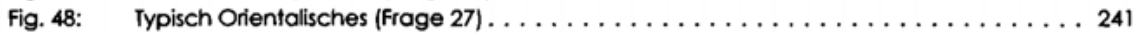

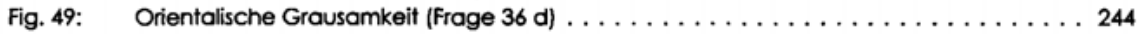

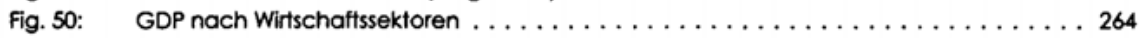

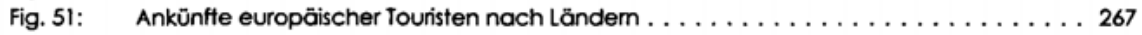




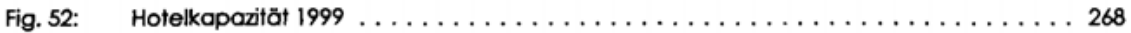

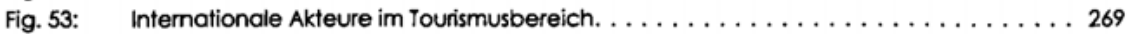

Fig. 54: Entführungen von Ausländern im Jemen (Personen) $\ldots \ldots \ldots \ldots \ldots \ldots \ldots \ldots \ldots$

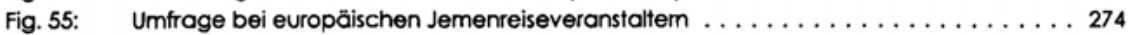

\section{Abblldungsverzeichnis}

Abb. 1

Abb. 2

Abb. 3

Abb. 4

Abb. 5:

Abb. 6

Abb. 7

Abb. 8

Abb. 9

Abb. 10

Abb. 11

Abb. 12

Abb. 13

Abb. 14

Abb. 15

Abb. 16

Abb. 17

Abb. 18

Abb. 19

Abb. 20

Abb. 21

Abb. 22

Abb. 23

Abb. 24

Abb. 25

Abb. 26

Abb. 27

Abb. 28

Abb. 38

Abb. 30

Abb. 31
Leviathan. Quelle: A. v. HARFF (1860). Kaffeepflanze. Quelle: P. ALPINO (1592) Kaffeepflanze. Quelle: J. DE LAROQUE (1740) Aden. Quelle: O. DAPPER (1681) Mocha. Quelle: F. PINTO MENDEI (1671). Mocha. Quelle: J. ANDERSEN (1669) Mocha. Quelle: O. DAPPER (1681) Mocha. Quelle: A. Etwood (1830) Kriegsübungen. C. NieBUHR (1772) Loheyya und Beit al Fakih. Quelle: C. NIEBUHR (1774) Yarim. Quelle: C. NIEBUHR (1774)

Taizz. Quelle: C. Niebuhr (1774) Sana'a. Quelle: C. Niebuhr (1774)

Taiz. Quelle: C. NIEBUHR (1774). Bergjemen. Quelle: C. NIEBUHR (1774)

Bergjemen. Quelle: C. NIEBUHR (1774) Imamsaudienz. Quelle: C. NiebuHr (1774)

Banian. Quelle: C. NieBUHR (1772) .

Araberin. Quelle: C. NIEBUHR (1774)

Araberin. Quelle: C. NieBuhr (1774) .

Araber. Quelle: C. NieBuhr (1774) .

Araber. Quelle: L. GrandPrÉ (1803)

Banian. Quelle: L. GrandPrE (1803)

Jemenit. Quelle: L. HIRSCH (1898)

Jemenit. Quelle: L. HIRSCH (1898)

SeEtzens Fund. Quelle: J. HAMMER-PurgSTALL (1811).

Weusteds Funde. Quelle: R. Weusted (1842)

Funde von HaLéYY. Quelle: HaLÉVY (1872)

Numismatik. Quelle: G. SCHLUMBERGER (1880) .

Sana'a. Quelle: F. Номmer (1883)

Bergdorf. Quelle: F. Номmer (1883)
Tafel 1-a Tafell-a Tafel t-a Tafel I-b Tafel l-c Tafel I-d Tafel -le Tafel -le Tafel II-a Tafel II-a Tafel II-b Tafel II-b Tafel II-C Tafel II-c Tafel II-c Tafel II-d Tafel II-d Tafel III -a Tafel III -a Tafel III -a Tafel III - a Tafel III -b Tafel III -b Tafel III -c Tafel III-d Tafel IV-a Tafel IV-a Tafel IV-a Tafel IV-b Tafel V-a Tafel V-a

\section{Kartenverzeichnis}

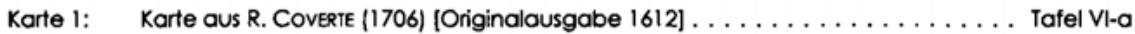

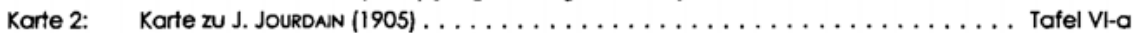
Karte 3: Karte aus S. BOCHART (1674) . . . . . . . . . . . . . . . . . . Tafel VI-a

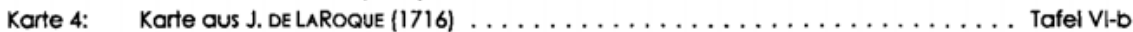
Karte 5: Karte aus R. WeuLTted $(1842) \ldots \ldots \ldots \ldots \ldots \ldots \ldots \ldots \ldots$ Tafel VI-b 